\title{
Lossless Data Hiding Technique using Reversible Function
}

\author{
Sang-Ho Shin ${ }^{1}$, Ho Hwang ${ }^{2}$ and Jun-Cheol Jeon ${ }^{2}$ \\ ${ }^{1}$ School of Computer Science and Engineering, Kyungpook National University \\ 80 Daehakro, Bukgu, Daegu, 702-701, Korea \\ ${ }^{2}$ Dept. of Computer Engineering, Kumoh National Institute of Technology \\ 61 Daehakro, Gumi, Gyeongbuk, 730-701, Korea \\ shshin80@infosec.knu.ac.kr, kcats9731@gmail.com,jcjeon@kumoh.ac.kr
}

\begin{abstract}
The most of the previous lossless data hiding techniques are that secret data are embedded into cover image. So, the relationship between the embedding capacity and PSNR of these techniques is always an inverse proportion. In contrast, the embedded position information of secret data are embedded into a location map in the proposed technique in order to achieve thedirectly proportional relationship. The proposed technique is based on a property of selfinverse in reversible function, it is the composite operation between reversible functions. In the embedding procedure, a stego image without distortion is generated using this property.In order to evaluate the efficiency and security of the proposed technique, the embedding capacity and PSNR are used in the experiments. In the experimental results, the embedding capacity and PSNR of the proposed technique are greater than it of the previous techniques.
\end{abstract}

Keywords: Lossless data hiding technique;reversible function;embedding capacity; PSNR

\section{Introduction}

With the growth of the Internet and computing technologies, data hiding, is the technique that secret data is hided in the meaningful host data in order to distract the attention of the observers [1-3], is attracting a lot of public attention. Besides, variety data in digital media such as text, image, audio and video files are being transmitted over the Internet. Many data hiding techniques were proposed [4-12].

Lossless data hiding is the technique that invisible data (which is called a secret) are embedded into a digital image (which is called a cover image).In this technique, a distortion on the image (which is called a stego image) after data embedding should be low. An advantage characteristic of lossless data hiding is reversibility, that is, the embedded data can remove from stego image in order to restore the original image. It can provide an authentication and integrity of a cover image. Secret data are embedded into a cover image in such away that an authorized party could extract the secret data, and also recover the original cover image [13-15].

The previous lossless data hiding techniques can be classified into two major categories: difference expansion (DE) and histogram shifting. Tian proposed a DE-based lossless data hiding technique for the first time $[14,15]$. A DE means that 1-bit secret data is embedded into an expanded difference within two consecutive pixel values in an image, and the difference is expanded by its binary representation and the addition of 1-bit secret data. An embedding capacity of his technique is close to 0.5 bit-per-pixel (bpp); but, there exists the significant distortion of stego image quality because bit-replacements of cover image pixels. Besides, this 
technique does not suitable for multiple embedding, which accumulates dramatic image

Table 1. The Truth Table of Tof foli Reversible Function

\begin{tabular}{c|c|c||c|c|c}
\hline \hline \multicolumn{3}{c||}{ Input } & \multicolumn{3}{c}{ Output } \\
\hline$A$ & $B$ & $C$ & $A^{\prime}$ & $B^{\prime}$ & $C^{\prime}$ \\
\hline \hline 0 & 0 & 0 & 0 & 0 & 0 \\
\hline 0 & 0 & 1 & 0 & 0 & 1 \\
\hline 0 & 1 & 0 & 0 & 1 & 0 \\
\hline 0 & 1 & 1 & 0 & 1 & 1 \\
\hline 1 & 0 & 0 & 1 & 0 & 0 \\
\hline 1 & 0 & 1 & 1 & 0 & 1 \\
\hline 1 & 1 & 0 & 1 & 1 & $\mathbf{1}$ \\
\hline 1 & 1 & 1 & 1 & 1 & $\mathbf{0}$ \\
\hline \hline
\end{tabular}

distortion within consecutive pixel values [16-20].

In the meantime, $\mathrm{Ni}$ et al. proposed a data hiding technique based histogram shifting [21]. Typical histogram is a graphical representation of the distribution of data. In this technique, it is acts as a graphical representation of the tonal distribution in a cover image. The histogram shifting is that secret data are embedded into the original peak pixel areas after the original peak pixel values is shifted by the direction of zero point. Ni et al.'s, scheme offers invisible image distortions with little auxiliary information; but, the embedding capacity is limited by the number of frequency of the peak pixels [22-26].

In this paper, we propose a new lossless data hiding technique without distortion of stego image. The proposed technique is based on a property of self-inverse in reversible function, it is the composite operation between reversible functions. In order to embed and extract a secret data, we constructed a pattern table using the property of self-inverse, and this table generated a stego image. Although a location map in embedding procedure is generated, distortion of stego image does not exist because the embedded position of secret data is embedded into location map. In the experimental results, the embedding capacity and PSNR of the proposed technique are greater than it of the previous techniques $[15,27]$.

This paper is organized as follows. Section 2 introduces reversible function concept. The proposed technique is discussed in Section 3. Section 4 analyzes the efficiency and security between the proposed and previous techniques. Finally, Section 5 gives the conclusions.

\section{Reversible Function}

An arbitrary function $f$ with domain $X$ and codomain $Y$ is commonly denoted by $f: X \rightarrow Y$. It is represented by $f: \mathbb{B}^{n} \rightarrow \mathbb{B}$ on Boolean algebra, where $\mathbb{B}$ is a set which consists of 1-bit as 0 or $1(\mathbb{B}=\{0,1\})$, and a set $\mathbb{B}^{n}$ consists of a $n$-bit $\left(\mathbb{B}^{n}=\{00 \ldots 0,00 \ldots 1, \ldots, 11 \ldots 1\}\right)$. On the other hand, a reversible function $f^{r}$ on Boolean algebra is denoted by $f^{r}: \mathbb{B}^{n} \rightarrow \mathbb{B}^{n}$. It is defined by Eq. (1). 


$$
f^{r}\left(b_{1}, b_{2}, \ldots, b_{n}\right)=c_{1}, c_{2}, \ldots, c_{n},
$$

where $b_{1}, b_{2}, \ldots, b_{n}$ and $c_{1}, c_{2}, \ldots, c_{n}$ are binary values and $c_{i}$ is represented by Eq. (2).

$$
c_{i}=f_{i}\left(b_{1}, \ldots, b_{i-1}, b_{i+1}, \ldots, b_{n}\right) \odot b_{i}
$$

where $f_{i}: \mathbb{B}^{n} \rightarrow \mathbb{B}$ is an arbitrary Boolean function and ' $\odot$ ' indicates Boolean algebra operator such as $A N D, O R, X O R$ or NOT. For example, Toffoli reversible function $f_{(\text {Tof foli })}^{r}: \mathbb{B}^{3} \rightarrow$ $\mathbb{B}^{3}$ can be represented by Eq. (3).

$$
f_{(\text {Toffoli })}^{r}\left(b_{1}, b_{2}, b_{3}\right)=c_{1}, c_{2}, c_{3},
$$

where $c_{1}, c_{2}$ and $c_{3}$ are $b_{1}, b_{2}$ and $f_{(A N D)}\left(b_{1}, b_{2}\right) \oplus b_{3}$ (where $f_{(A N D)}\left(b_{1}, b_{2}\right)=b_{1} \wedge b_{2}, ' \wedge$ ' and ' $\oplus$ ' indicate an $A N D$ and $X O R$ ), respectively. The truth table of this function is shown in Table 1. Typical function $f$ takes $n$ inputs and generates a single output; the reversible function $f^{r}$ takes $n$ inputs and produces $n$ outputs.

It has some properties as follows. For any general function $f: X \rightarrow Y$, an inverse function $f^{-1}$ should be required in order to perform an arithmetic operation $Y \rightarrow X$. Also, $f$ should be an one-to-one mapping between domain and codomain elements so that there exists an inverse function $f^{-1}$. But, a reversible function $f^{r}$ can operate $f^{r}: X \rightarrow Y$ and $f^{r}: Y \rightarrow X$. This is because it perform a self-inverse and this fact can be represented by Eq. (4).

$$
f \circ f^{-1}=f^{r} \circ\left(f^{r}\right)^{-1}=f^{r} \circ\left(f^{r} \circ \cdots \circ f^{r}\right)=I,
$$

where' $\circ$ ' and $I$ indicate a composite arithmetic operation between reversible functions and an identity function, respectively. That is, $f^{r} \circ \cdots \circ f^{r}$ can act as an inverse function $f^{-1}$. All reversible functions can be represented as a matrix form because the number of inputs and outputs is the same. For example, Toffoli reversible function [28] is represented as a matrix form by Eq. (5). And it can perform a self-inverse as shown in Eq. (6). In this paper, we propose a lossless data hiding technique based on properties of reversible function.

$$
\begin{aligned}
& f_{(\text {Toffoli })}^{r}=\left[\begin{array}{llllllll}
1 & 0 & 0 & 0 & 0 & 0 & 0 & 0 \\
0 & 1 & 0 & 0 & 0 & 0 & 0 & 0 \\
0 & 0 & 1 & 0 & 0 & 0 & 0 & 0 \\
0 & 0 & 0 & 1 & 0 & 0 & 0 & 0 \\
0 & 0 & 0 & 0 & 1 & 0 & 0 & 0 \\
0 & 0 & 0 & 0 & 0 & 1 & 0 & 0 \\
0 & 0 & 0 & 0 & 0 & 0 & 0 & 1 \\
0 & 0 & 0 & 0 & 0 & 0 & 1 & 0
\end{array}\right] \\
& f_{(\text {Toffoli })}^{r} \circ f_{(\text {Toffoli })}^{r}=\left[\begin{array}{llllllll}
1 & 0 & 0 & 0 & 0 & 0 & 0 & 0 \\
0 & 1 & 0 & 0 & 0 & 0 & 0 & 0 \\
0 & 0 & 1 & 0 & 0 & 0 & 0 & 0 \\
0 & 0 & 0 & 1 & 0 & 0 & 0 & 0 \\
0 & 0 & 0 & 0 & 1 & 0 & 0 & 0 \\
0 & 0 & 0 & 0 & 0 & 1 & 0 & 0 \\
0 & 0 & 0 & 0 & 0 & 0 & 0 & 1 \\
0 & 0 & 0 & 0 & 0 & 0 & 1 & 0
\end{array}\right]\left[\begin{array}{llllllll}
1 & 0 & 0 & 0 & 0 & 0 & 0 & 0 \\
0 & 1 & 0 & 0 & 0 & 0 & 0 & 0 \\
0 & 0 & 1 & 0 & 0 & 0 & 0 & 0 \\
0 & 0 & 0 & 1 & 0 & 0 & 0 & 0 \\
0 & 0 & 0 & 0 & 1 & 0 & 0 & 0 \\
0 & 0 & 0 & 0 & 0 & 1 & 0 & 0 \\
0 & 0 & 0 & 0 & 0 & 0 & 0 & 1 \\
0 & 0 & 0 & 0 & 0 & 0 & 1 & 0
\end{array}\right]=I(6)
\end{aligned}
$$

\section{The Proposed Technique}

In this section, we discuss the main concept, the embedding and extraction algorithms of the proposed technique. 
Table 2. The Example of a Pattern Table for $\boldsymbol{f}_{(N O T)}^{r} \circ \boldsymbol{f}_{(N O T)}^{r}$

\begin{tabular}{c|c|c}
\hline \hline \multirow{2}{*}{$\begin{array}{c}\text { Case } \\
(\bmod 4)\end{array}$} & $\begin{array}{c}\text { A pixel in } \\
\text { cover image }\end{array}$ & $\begin{array}{c}\text { A pixel in } \\
\text { stego image }\end{array}$ \\
\cline { 2 - 3 } & $L S B_{2}$ & $L S B_{2}$ \\
\hline \hline $1\left(=01_{(2)}\right)$ & 00 & 00 \\
\hline $2\left(=10_{(2)}\right)$ & 01 & 01 \\
\hline $0\left(=00_{(2)}\right)$ & 10 & 10 \\
\hline $3\left(=11_{(2)}\right)$ & 11 & 11 \\
\hline \hline
\end{tabular}

\subsection{The Main Concept}

Unlike previous lossless data hiding techniques is that secret data are directly embedded into cover image, the embedded positions of secret data are embedded into a location map in the proposed technique. A location map means a set of information for position of embedded secret data, and it is the same size of cover image. Also, a pattern table is constructed using a property of self-inverse in reversible function and, an advantage of this table is that distorionless stgeo image is generated. This is because a pattern table is based on an identity function which consists composite operation between reversible functions as shown in Eq. (4).

Given that reversible function $f^{r}: \mathbb{B}^{2} \rightarrow \mathbb{B}^{2}$, for example, a $f_{(N O T)}^{r}$ is represented by Eq. (7).

$$
f_{(\text {NOT })}^{r}\left(b_{1}, b_{2}\right)=c_{1}, c_{2},
$$

where $c_{1}$ and $c_{2}$ are $b_{2}$ and $b_{1}$, respectively. And it can be expressed as a matrix form by Eq. (8).

$$
f_{(N O T)}^{r}=\left[\begin{array}{ll}
0 & 1 \\
1 & 0
\end{array}\right]
$$

$f_{(N O T)}^{r}$ is satisfied by Eq. (9).

$$
f_{(N O T)}^{r} \circ f_{(N O T)}^{r}=\left[\begin{array}{ll}
0 & 1 \\
1 & 0
\end{array}\right]\left[\begin{array}{ll}
0 & 1 \\
1 & 0
\end{array}\right]=I
$$

So, we can construct a pattern table as shown in Table 2. In Table 2,LSB $B_{2}$ indicates least significant bit(LSB) two bits of a pixel value in an image. And the meaning of "case $(\bmod 4)$ " is that an $i$-th pixel value $\left(S_{i}\right)$ in a secret image $(S I)$ is applied by modulo arithmetic operation.For $f_{(N O T)}^{r} \circ f_{(N O T)}^{r}$, the number of cases of secret is four such as $0\left(=00_{(2)}\right)$, $1\left(=01_{(2)}\right), 2\left(=10_{(2)}\right)$ and $3\left(=11_{(2)}\right)$, and the order of these is " $\cdots \rightarrow 1 \rightarrow 2 \rightarrow 0 \rightarrow$ $3 \rightarrow 1 \rightarrow \cdots$. The number of orders is $4 !(=24)$.

The example of the embedding procedure is as follows. Given that an $i$-th pixel value $C_{i}$ of cover image $(C I)$, a location map which is the same size of $C I$, a $j$-th secret $s_{j}$ and a pattern table for $f_{(N O T)}^{r} \circ f_{(N O T)}^{r}$ as shown in Table 2, if $L S B_{2}$ of $C_{i}(=124)$ is 00 and $s_{j}=1$, it corresponds first case. So, $i$-th pixel value $S T_{i}$ of stego image (STI) which is the same value as a $C_{i}$ is generated. And then, $i$-thindex in a location map is written by 1 . The meaning of ' 1 ' in location map is that secret $s_{j}$ is embedded into $C I$. On the other hand, if a pair of $L S B_{2}$ of $C_{i}$ and $s_{j}$ do not correspond in the pattern table, this case does not perform the embedding. Also, corresponding index in a location map is written by 0 . Other pixels in cover image are repeated at the same method. Lastly, a pattern table and location map through secure channel are transmitted. 


\subsection{The Embedding Algorithm}

Input: a $C I$ with size of $M \times M$ and a $S I$ with size of $N \times N$

Output: $S T I$ with size of $M \times M$, a pattern table and a location map

Step 1: Choose a positive integer $n$ in order to determine the number of inputs and outputs of reversible function $f^{r}: \mathbb{B}^{n} \rightarrow \mathbb{B}^{n}$ and calculate $m=2^{n}$, where $m$ indicates the number of cases of secret image, and the other meaning is the number of reversible functions asm $=\left|f^{r}\right|$.

Step2: Choose a reversible function $f^{r}: \mathbb{B}^{n} \rightarrow \mathbb{B}^{n}$ of $m$ cases. And then, construct apattern table of $f_{(\text {chosen })}^{r}: \mathbb{B}^{n} \rightarrow \mathbb{B}^{n}$ as follows.

Step 2.1: Choose the number of $L S B \mathrm{~s}(|L S B|)$ for each pixel within divisors of $n$. According to the number of $L S B \mathrm{~s}$, the number of required pixels in $C I$ is determined as $m /|L S B|$ (where $|L S B|$ is always even).

Step 2.2: Choose the number of cases of secret within divisors of $m$ (where the number of cases is more than 1 ).

Step 3: Convert an $i$-th pixel value $\left(S_{i}\right)$ in $S I$ into $m$-ary's expression as Eq. (10).

$$
S_{i} \Rightarrow\left\{s_{i}\left\lceil\log _{m} 255\right\rceil, \ldots, s_{(i+1)}\left\lceil\log _{m} 255\right\rceil-1\right\},
$$

where $1 \leq i \leq N^{2}-1$. Let a set $\mathbb{S}$ consists of $\left(N^{2}\right)\left\lceil\log _{m} 255\right\rceil$ elements that compose of $m$-ary's values, and it is expressed by Eq. (11).

$$
\mathbb{S}=\left\{s_{0}, \ldots, s_{\left(N^{2}\right)\left[\log _{m} 255\right]-1}\right\}
$$

Step 4: Embed a $j$-th $\left(0 \leq j \leq\left(N^{2}\right)\left\lceil\log _{m} 255\right\rceil-1\right)$ secret into a $i$-th pixel value in a cover image with the pattern table, if $j$-th secret and $L S B_{2}$ of $i$-th pixel value are corresponded. Otherwise, the embedding does not perform. If a secret bit is embedded into cover image, 1 is represented at corresponding pixel index in location map. Otherwise, 0 is represented. If the embedding procedure is completed, a stego image is generated, and then apattern table of reversible function and location map through secure channel are transmitted.

\subsection{The Extraction Algorithm}

Input: $S T I$ with size of $M \times M$, a pattern table and a location map

Output: a recovered $C I(R C I)$ with size of $M \times M$ and a recovered $S I(R S I)$ with size of $N \times N$

Step 1: Extract a $j$-th $\left(0 \leq j \leq\left(N^{2}\right)\left\lceil\log _{m} 255\right\rceil-1\right)$ secret from $i$-th $\left(0 \leq i \leq M^{2}-1\right)$ pixel value in a stego image.Given that a location map and the pattern table, if $i$-th value of a location map is 1 , corresponding secret $s_{j}$ and cover image pixel value $C_{i}$ are extracted from $S T_{i}$ by the pattern table. Otherwise, a cover image pixel value $C_{i}$ are extracted from $S T_{i}$ by the pattern table.

Step 2: Convert the calculated $\mathrm{s}_{0}, \ldots, \mathrm{s}_{\left(\mathrm{N}^{2}\right)\left\lceil\log _{\mathrm{m}} 255\right]-1}$ into pixel values by $m$. And then, they are reconstructed by a form of $R S I$ and $R C I$. 
Table 3. The Example of a Pattern Table for $f^{r}: \mathbb{B}^{1} \rightarrow \mathbb{B}^{1}$

\begin{tabular}{c|c|c}
\hline \hline \multirow{2}{*}{$\begin{array}{c}\text { Case 1 } \\
\bmod 2)\end{array}$} & $\begin{array}{c}\text { A pixel in } \\
\text { cover image }\end{array}$ & $\begin{array}{c}\text { A pixel in } \\
\text { stego image }\end{array}$ \\
\cline { 2 - 3 } & $L S B_{1}$ & $L S B_{1}$ \\
\hline \hline $1\left(=1_{(2)}\right)$ & 0 & 0 \\
\hline $0\left(=0_{(2)}\right)$ & 1 & 1 \\
\hline \hline
\end{tabular}

\section{The Experimental Results}

In this section, the embedding capacity and PSNR of the proposed scheme are analyzed.

\subsection{The Measurement Tools}

In order to evaluate the efficiency and security of data hiding technique, there exist two typical measurement tools: the embedding capacity and PSNR. The embedding capacity meansthe amount of embedded secret data in a cover image, and it can evaluate the efficiency of data hiding technique. That is, if the embedding capacity of an arbitrary technique is more increased, we can say that this technique has a good efficiency. It is generally measured in bit-per-pixel (bpp) or bit.

PSNR is the abbreviation for "peak signal-to-noise ratio" and it is the ratio between the maximum possible power of a signal and the power of corrupting noise that affects the fidelity of its representation. Nowadays, PSNR is the most popular distortion measurement tool in the field of image and video coding and compression. It is usually measured in decibels $(d B)$, and well known that these difference distortion metrics are not very well correlated with the human visible system (HVS). This might be a problem for their application in secret image since sophisticated data hiding techniques exploit in one way or the other effects of these schemes [1][2]. The detailed PSNR is represented by Eq.(12).

$$
P S N R=10 \log \left(M A X^{2} / M S E\right),
$$

where $M A X$ is the maximum value that a pixel can be represented. It is 255 because greyscale test images were used in this paper. $M S E$ is the abbreviation for "mean squared error" and it is represented by Eq.(13).

$$
M S E=\frac{1}{M^{2}} \sum_{i=0}^{M^{2}-1}\left(C_{i}-S T_{i}\right)^{2},
$$

where $M$ indicates the size of $C I$ and $S T I . C_{i}$ and $S T_{i}$ are $i$-th pixel values in $C I$ and $S T I$, respectively. Given that two grey-scale images, if $P S N R$ value is close to infinity $(=\infty)$, the distortion between two images is zero, that is, two images are the same.On the other hand, if $P S N R$ value is close to zero, the distortion is higher, that is, two images are different. Generally, $P S N R$ value is more that $35 d B$, the difference between two images cannot distinguish in HVS.If the distortion for an arbitrary technique is close to zero, we can say that the security of this technique is a good [1].

\subsection{Analysis of Efficiency and Security}

In the experiments, we used eight grey-scale test images as shown in Figure 2. The sizes of $C I$ and STIare $512 \times 512$. The secret data was generated by Rand function in C++ Library. And then, generated secret bitstream was composed by each eight-bit. In order to implement the proposed scheme, the OpenCV Library and $\mathrm{C}++$ programming language were used. Also, we 


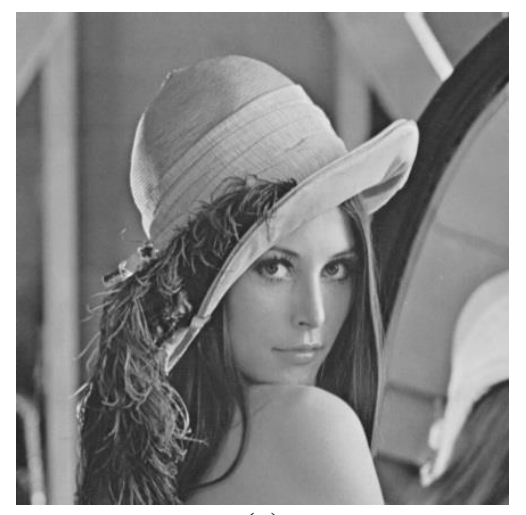

(a)

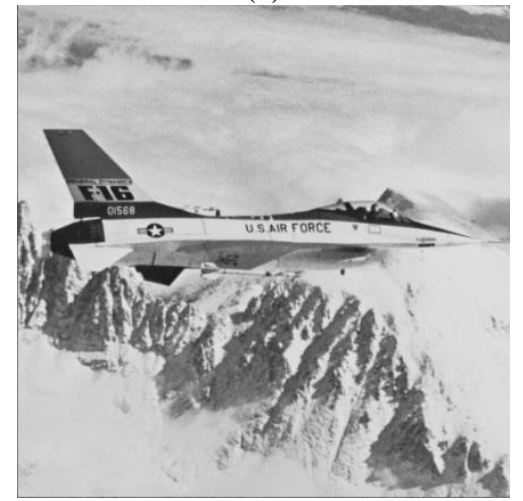

(c)

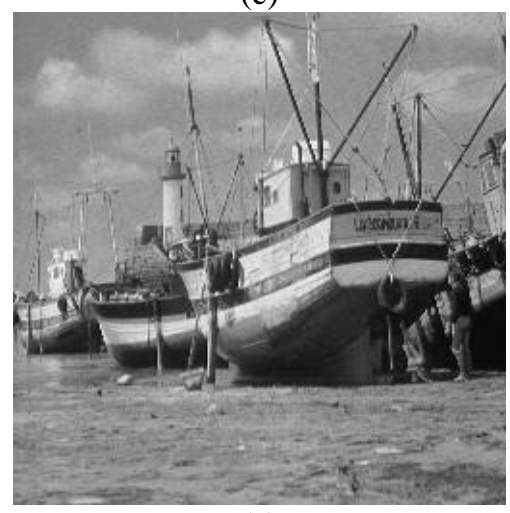

(e)

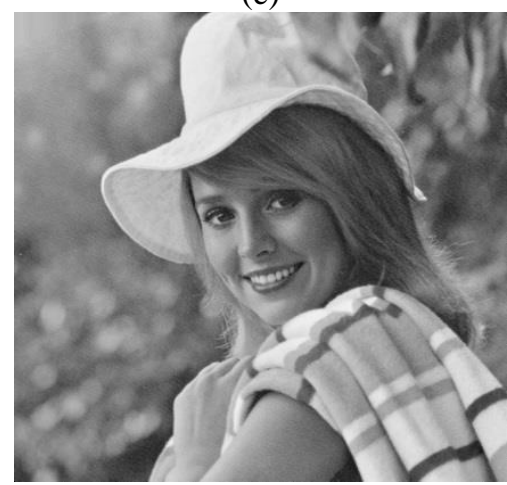

(g)

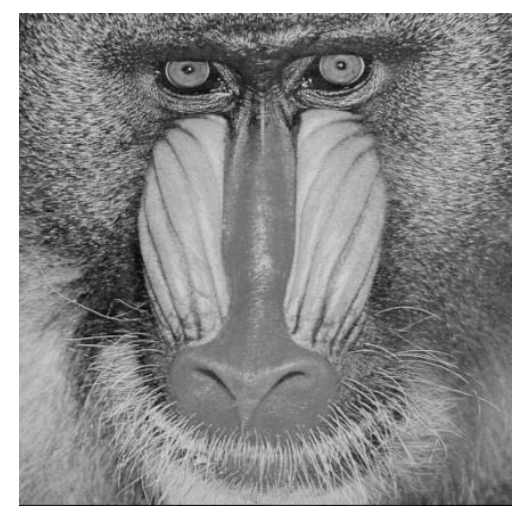

(b)

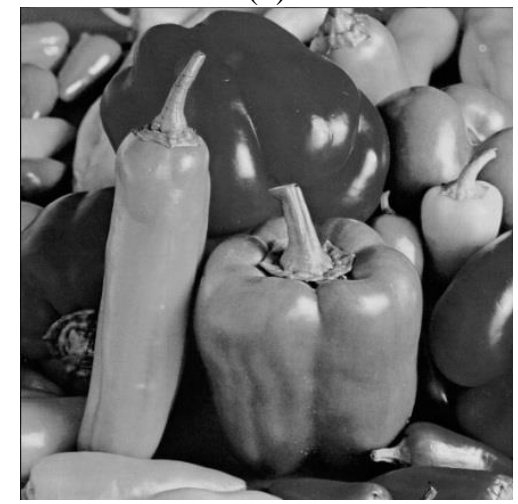

(d)

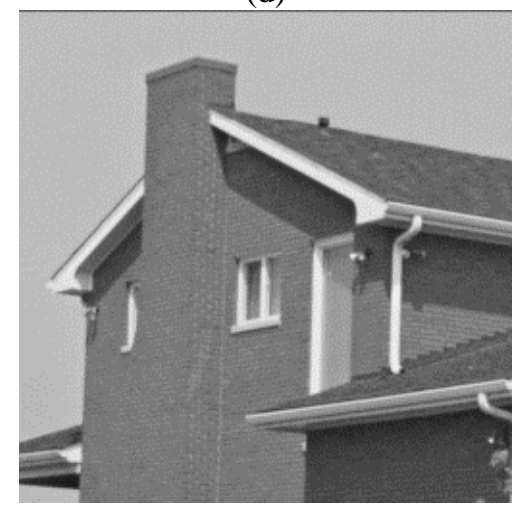

(f)
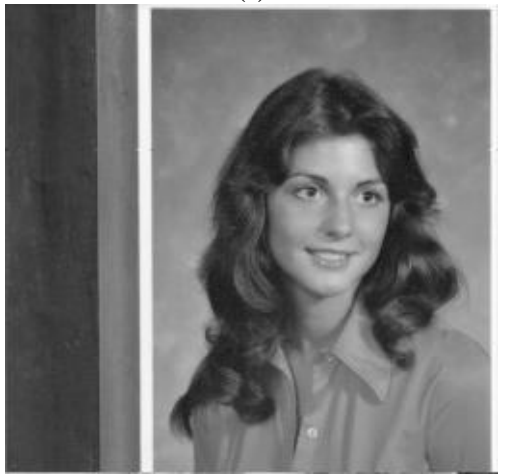

(h)

Figure 1. Eight Grey-scale Test Images 
Table 4. The Example of a Pattern Table for $f^{r}: \mathbb{B}^{2} \rightarrow \mathbb{B}^{2}$

\begin{tabular}{c|c|c|c}
\hline \hline $\begin{array}{c}\text { Case 2 } \\
(\bmod 2)\end{array}$ & $\begin{array}{c}\text { Case 3 } \\
(\bmod 4)\end{array}$ & $\begin{array}{c}\text { A pixel in } \\
\text { cover } \\
\text { image }\end{array}$ & $\begin{array}{c}\text { A pixel in } \\
\text { stego } \\
\text { image }\end{array}$ \\
\cline { 3 - 4 } & $L S B_{2}$ & $L S B_{2}$ \\
\hline \hline $1\left(=1_{(2)}\right)$ & $3\left(=11_{(2)}\right)$ & 00 & 00 \\
\hline $0\left(=0_{(2)}\right)$ & $1\left(=01_{(2)}\right)$ & 01 & 01 \\
\hline $0\left(=0_{(2)}\right)$ & $0\left(=00_{(2)}\right)$ & 10 & 10 \\
\hline $1\left(=1_{(2)}\right)$ & $2\left(=10_{(2)}\right)$ & 11 & 11 \\
\hline \hline
\end{tabular}

Table 5. Result of the Embedding Capacity of the Proposed and Previous Techniques

\begin{tabular}{l||c|c||c|c|c}
\hline \hline \multirow{2}{*}{$\begin{array}{l}\text { Test } \\
\text { images }\end{array}$} & \multirow{2}{*}{$\begin{array}{c}\text { Difference } \\
\text { expansion } \\
\text { (bits) }\end{array}$} & $\begin{array}{c}\text { Histogram } \\
\text { Shifting } \\
\text { (bits) }\end{array}$ & $\begin{array}{c}\text { Case 1 } \\
\text { (bits) }\end{array}$ & $\begin{array}{c}\text { Case 2 } \\
\text { (bits) }\end{array}$ & $\begin{array}{c}\text { Case 3 } \\
\text { (bits) }\end{array}$ \\
\cline { 5 - 7 } Lena & 39,566 & 47,201 & 65,524 & 131,680 & 130,906 \\
\hline \hline Baboon & 34,256 & 18,533 & 65,576 & 130,068 & 130,301 \\
\hline Airplane & 40,657 & 30,631 & 65,456 & 131,014 & 131,230 \\
\hline Pepper & 39,824 & 35,155 & 65,112 & 132,524 & 133,766 \\
\hline Boat & 39,257 & 29,210 & 65,425 & 130,778 & 130,715 \\
\hline Man & 39,447 & 40,748 & 67,276 & 130,578 & 128,837 \\
\hline House & 40,002 & 35,868 & 66,782 & 130,852 & 123,277 \\
\hline Woman & 39,872 & 37,602 & 68,258 & 130,430 & 131,238 \\
\hline \hline
\end{tabular}

have performed the experiments with examples of $f^{r}: \mathbb{B}^{1} \rightarrow \mathbb{B}^{1}$ and $f^{r}: \mathbb{B}^{2} \rightarrow \mathbb{B}^{2}$ as shown in Table 3 and 4, respectively.

In the proposed technique, if the embedding algorithm with $f^{r}: \mathbb{B}^{1} \rightarrow \mathbb{B}^{1}$ and a cover image (size is $M \times M$ ) performs one round, the embedding capacity is roughly $M^{2} / 4$ bitsdue to the pattern table. If a secret and $L S B$ pair of cover and stego pixels are 1 and 00 in Table 3, for example, the embedding can be performed. But, the embedding dose not perform the other $L S B$ pairs such as 01,10 and 11 . That is, only one case of four $L S B$ pairs is embedded. So, the probability of embedding is $1 / 4$. Hence, the embedding capacity is roughly $M^{2} / 4$ bits in one round.If the embedding algorithm with $f^{r}: \mathbb{B}^{2} \rightarrow \mathbb{B}^{2}$ and a cover image (size is $M \times M$ ) performs one round, the embedding capacity is roughly $M^{2} / 2$ bits by the same probability logic.The result of the embedding capacity between the proposed and previous techniques $[15,27]$ is shown in Table 5. In Table 5, the embedding capacity of the proposed technique is greater than $\mathrm{DE}$ and histogram shifting techniques by 1.6 and 1.8 times, respectively. The size of $C I$ is $512 \times 512(=262,144)$, and the embedding capacity of 'case 1' is roughly 65,536 bits in stochastic approach. The results of 'case 1' are close to 65,536 bits. Also, the results of 'case 1' and 'case 2' are close to 131,072 bits. 
Table 6. Result of PSNR of the Proposed and Previous Techniques

\begin{tabular}{l|c|c|c|c|c}
\hline \hline \multirow{2}{*}{$\begin{array}{l}\text { Test } \\
\text { images }\end{array}$} & \multirow{2}{*}{$\begin{array}{c}\text { Difference } \\
\text { expansion }\end{array}$} & $\begin{array}{c}\text { Histogram } \\
\text { Shifting }\end{array}$ & \multicolumn{3}{|c}{ Proposed technique } \\
\cline { 5 - 6 } Lena & 44.20 & 48.54 & $\infty$ & $\infty$ & $\infty$ \\
\hline Baboon & 42.82 & 48.29 & $\infty$ & $\infty$ & $\infty$ \\
\hline Airplane & 43.54 & 48.39 & $\infty$ & $\infty$ & $\infty$ \\
\hline Pepper & 43.25 & 48.44 & $\infty$ & $\infty$ & $\infty$ \\
\hline Boat & 43.84 & 48.38 & $\infty$ & $\infty$ & $\infty$ \\
\hline Man & 44.56 & 48.56 & $\infty$ & $\infty$ & $\infty$ \\
\hline House & 43.47 & 48.44 & $\infty$ & $\infty$ & $\infty$ \\
\hline Woman & 43.88 & 48.57 & $\infty$ & $\infty$ & $\infty$ \\
\hline \hline
\end{tabular}

Table 7. Result of Multi Rounds Embedding of the Proposed and Previous Techniques for Lena Grey-Scale Image

\begin{tabular}{|c|c|c|c|c|c|c|c|}
\hline \multirow{2}{*}{\multicolumn{2}{|c|}{ Techniques }} & \multirow{2}{*}{ Tools } & \multicolumn{5}{|c|}{ Round } \\
\hline & & & 1 & 2 & 3 & 4 & 5 \\
\hline \multirow{2}{*}{\multicolumn{2}{|c|}{$\begin{array}{l}\text { Difference } \\
\text { expansion }\end{array}$}} & $E C$ & 39,566 & 63,676 & 84,066 & 101,089 & 120,619 \\
\hline & & PSNR & 44.20 & 42.86 & 41.55 & 40.06 & 37.66 \\
\hline \multirow{2}{*}{\multicolumn{2}{|c|}{ Histogram shifting }} & $E C$ & 47,201 & 60,293 & 94,372 & 110,100 & 123,208 \\
\hline & & PSNR & 48.54 & 43.74 & 40.82 & 37.56 & 36.25 \\
\hline \multirow{2}{*}{ Proposed } & \multirow{2}{*}{ Case 2} & $E C$ & 131,680 & 263,421 & 526,952 & $1,053,893$ & $2,107,807$ \\
\hline & & PSNR & $\infty$ & $\infty$ & $\infty$ & $\infty$ & $\infty$ \\
\hline
\end{tabular}

The distortion of stego image was naturally occurred because secret data were directly embedded into cover image in the previous techniques [15][27]. But, the distortion does not exist stego image in the proposed technique because the embedded position in $C I$ of secret data was embedded into a location map. Also, cover and stego images are the same due to using the inverse property of reversible function. So, PSNR result of our technique is infinity as shown in Table 6.

One of advantages for typical lossless data hiding techniques is that the even if an embedding procedure performs several times, the result of PSNR is good as shown in Table 7. In Table 7, if an embedding procedure performs five times, the embedding capacity of the proposed technique are greater than DE and histogram shifting technique by 17.4 and 17.1 times. Moreover, PSNR of the previous techniques is progressively decreased, it is always infinity in our technique. 


\section{Conclusions}

In this paper, we proposed a new lossless data hiding technique without distorition. The proposed technique is based on a reversible function, and it has a property of selfinverse which is composite operation between reversible functions. In order to embed and extract a secret data, we constructed a pattern table using the property of selfinverse, and this table generated a stego image. Although a location map was generated, distortion of stego image does not exist because the embedded position of secret data was embedded into location map. That is, secret data does not embed into cover image, directly. In the experimental results, the embedding capacity and PSNR of the proposed technique were greater than it of the previous techniques.

\section{Acknowledgement}

This research was supported by Basic Science Research Program through the National Research Foundation of Korea(NRF) funded by the Ministry of Science, ICT \& Future Planning (2011-0014977).

\section{References}

[1] S. Katzenbeisser and F. A. P. Petitcolas, "Information hiding techniques for steganography and digital watermarking”, Norwood: Artech house, (2000).

[2] I. J. Cox, M. L. Miller, J. A. Bloom, J. Fridrich and T. Kalker, "Digital watermarking and steganography", Morgan Kaufmann, (2007).

[3] A. J. Menezes, P. C. Van Oorschot and A. Scott, "Handbook of Applied Cryptography", CRC press, BocaRaton, FL, (1997).

[4] J.-C. Jeon, "Analysis of Hash Functions and Cellular Automata Based Schemes", International Journal of Security and Its Applications, vol. 7, no. 3, (2013).

[5] V. Saxena, A. Harsulkar, P. Khemka and J. P. Gupta, "Performance Analysis of Color Channel for DCT Based Image Watermarking Scheme”, International Journal of Security and its Applications, vol. 1, no. 2, (2007).

[6] B. Vijaya Kumar, M. Radhika Mani and G. RoselineNesaKumari, "A New Marginal Color Image Water Marking Method based on Logical Operators", International Journal of Security and its Applications, vol. 3, no. 4, (2009).

[7] N. Tiwari and M. Shandilya, "Secure RGB Image Steganography from Pixel Indicator to Triple AlgorithmAn Incremental Growth", International Journal of Security and its Applications, vol. 4, no. 4, (2010).

[8] A. Cheddad, J. Condell, K. Curran and P. McKevitt, "Digital image steganography: Survey and analysis of current methods", Signal Processing, vol. 90, no. 3, (2010).

[9] S. H. Low, N. F. Maxemchuk, J. T. Brassil and L. O. Gorman, "Document marking and identification using both line and word shifting", Proceedings of the FourteenthAnnual Joint Conference of the IEEE Computer and Communications Societies, (1995) April 2-6.

[10] K. Fopalan, "Audio steganography using bitmodification", Proceedings of the IEEE international Conference on Acoustics, Speech, and Signal processing, (2003) April6-10.

[11] M. Iwata, K. Miyake and A. Shiozaki, "Digital Steganography Utilizing Features of JPEGImages", IEICE trans, Fundamentals. E87-A, (2004).

[12] H. C. Wu, N. I. Wu, C. S. Tsai and M. S. Hwang, "Image Steganographic Scheme Based on Pixel-value Differencing and LSB Replacement Methods”, IEE Proceedings Vision, Image \& Signal Processing, vol. 152, no. 5, (2005).

[13] J. Fridrich, M. Goljan and R. Du, "Lossless data embedding-new paradigm in digital watermarking", Special Issue on Emerging Applications of Multimedia Data Hiding, vol. 2, (2002).

[14] T. Jun, "Reversible watermarking by difference expansion", Proceedings of workshop on multimedia and security, (2002).

[15] J. Tian, "Reversible Data Embedding Using a Difference Expansion, Circuits and Systems for Video Technology", IEEE Transactions on, vol. 13, no. 8, (2003).

[16] A. M. Alattar, "Reversible watermark using the difference expansion of ageneralized integer transform", IEEE Transactions on Image Processing, vol. 13, no. 8, (2004). 
[17] M. Carli, P. Campisi, and A. Neri, "Perceptual aspects in data hiding", Telecommunication Systems, vol. 33, (2006).

[18] M. U. Celik, G. Sharma, A. M. Tekalp and E. Saber, "Lossless generalized-LSB data Embedding", IEEE Transactions on Image Processing, vol. 14, no. 2, (2005).

[19] C. C. Chang, Lu and T. C., "A difference expansion oriented data hiding scheme for restoring the original host images", Journal of Systems and Software, vol. 79, no. 12, (2006).

[20] C. C. Lee, H. C. Wu, C. S. Tsai, and Y. P. Chu, "Adaptive lossless steganographic scheme with centralized difference expansion”, Pattern Recognition, vol. 41, no. 6, (2008).

[21] Z. Ni, Y.-Q. Shi, N. Ansari and W. Su, "Reversible data hiding, Circuits and Systems for Video Technology", IEEE Transactions, vol. 16, no. 3, (2006).

[22] C.-F. Lee and H.-L. Chen, "A novel data hiding scheme based on modulus function", Journal of Systems and Software, vol. 83, no. 5, (2010).

[23] J.-D. Lee, Y.-H. Chiou and J.-M. Guo, "Reversible data hiding based on histogram modification of SMVQ indices", Information Forensics and Security, IEEE Transactions on, vol. 5, no. 4, (2010).

[24] I.-C. Lin, Y.-B. Lin and C.-M. Wang, "Hiding data in spatial domain images with distortion tolerance", Computer Standards \& Interfaces, vol. 31, no. 2, (2009).

[25] H. Luo, F.-X. Yu, H. Chen, Z.-L. Huang, H. Li and P.-H. Wang, "Reversible data hiding based on block median preservation", Information Sciences, vol. 181, no. 2, (2011).

[26] W.-L. Tai, C.-M. Yeh and C.-C. Chang, "Reversible data hiding based on histogram modification of pixel differences", Circuits and Systems for Video Technology, IEEE Transactions on, vol. 19, no. 6, (2009).

[27] Y.-C. Li, C.-M. Yeh and C.-C. Chang, "Data hiding based on the similarity between neighboring pixels with reversibility”, Digital Signal Processing, vol. 20, no. 4, (2010).

[28] D. McMahon, "Quantum computing explained”, Wiley, (2007).
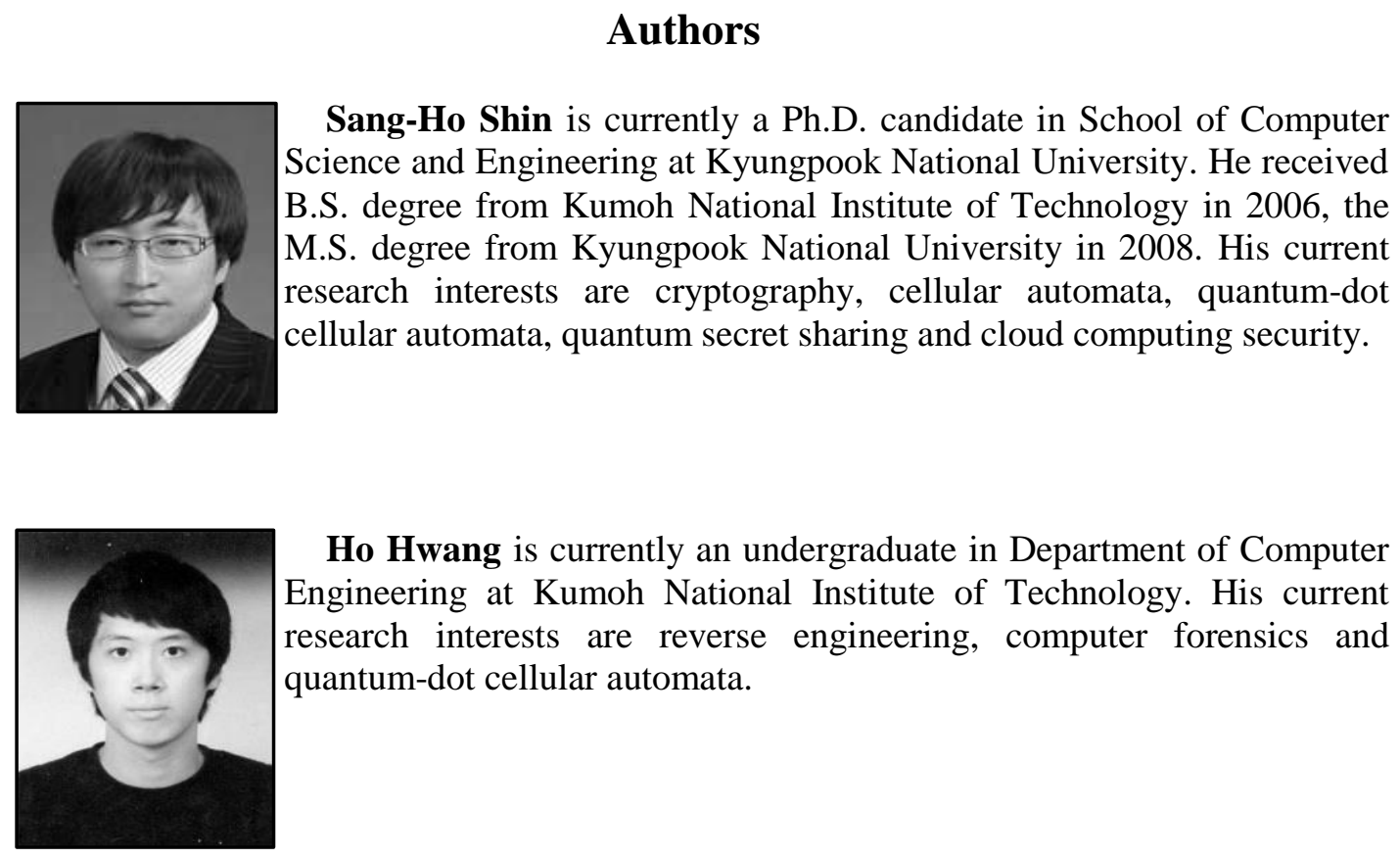

Ho Hwang is currently an undergraduate in Department of Computer Engineering at Kumoh National Institute of Technology. His current research interests are reverse engineering, computer forensics and quantum-dot cellular automata.

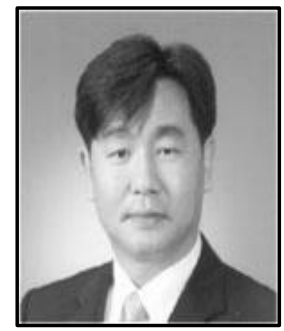

Jun-CheolJeon is currently a professor in Department of Computer Engineering at Kumoh National Institute of Technology. He received B.S. degree from Kumoh National Institute of Technology in 2000, the M.S. and Ph.D. degrees from Kyungpook National University in 2003 and 2007 respectively. His current research interests are cryptography, cellular automata, quantum-dot cellular automata and quantum computation. 
International Journal of Security and Its Applications Vol.8, No.1 (2014) 